\title{
Nuevos retos para la revista Adicciones: hacia la internacionalización de la revista y la mejora de su calidad
}

\author{
Amador Calafat*, Elisardo Becoña**, Julio Bobes** \\ * Director de Adicciones \\ **Editores Asociados \\ Enviar correspondencia: \\ Amador Calafat. Adicciones. Rambla, 15, 2º $3^{a} .07003$ Palma de Mallorca (España) \\ adicciones@sociodrogalcohol.org
}

\section{RESUMEN}

La revista Adicciones lleva 15 años publicándose. Se ha convertido en la revista de referencia en el tema de adicciones en habla hispana. Se trata de un proyecto editorial consolidado, pero no obstante quedan retos por plantearse como pueden ser entrar en algunas bases de datos relevantes como Medline o Current contents, tras haber conseguido entrar ya en Psychinfo que es la base de datos más importante en psicología, gestionada por la American Psychological Association.

No estar en estas bases de datos genera una situación viciada nada fácil de resolver pues hay autores que prefieren, por cuestiones académicas o de otro tipo, enviar sus trabajos a revistas inglesas con factor de impacto, con lo cual a su vez estas revistas siguen aumentando su importancia y su distancia respecto a las no incluidas en la base que provee el factor de impacto. Interesa pues un compromiso de todos (autores, revisores y editores) para elevar el nivel de la revista y conseguir un mayor reconocimiento internacional.

Adicciones da pasos en conseguir este reconocimiento no sólo trabajando dentro de ISAJE (International Society of Addiction Journal Editors), sino sobre todo comprometiéndose en una línea de mejora de los aspectos formales y de calidad de los artículos que publica, haciendo más claras y precisas las normas de publicación, siguiendo escrupulosamente los acuerdos de Farmington y renovando y ampliando su comité editorial.

Palabras clave: revistas, adicciones, factor de impacto, proceso editorial.

\section{ABSTRACT}

The Adicciones Journal has been published for 15 years during which time it has become the journal of reference on the subject of addictions in the Spanish language. Although it is well established editorially, there are still challenges to be faced such as its inclusion in certain relevant databases such as Medline or Current Contents, following its inclusion in Psychinfo, the most important database in the field of psychology, managed by the American Psychological Association

Not being included in these databases generates a vicious circle that is not easy to resolve as there are authors who, for academic or other reasons, prefer to publish their work in English language journals with a wider impact so that these journals, as a result, continue to expand their importance and scope in comparison with those not included in the database responsible for this very impact factor. The commitment of all (authors, revisers and editors) in raising the level of the Journal, and in ensuring greater international recognition, is essential in raising the level of the Journal and achieving greater international recognition.

Adicciones is taking steps to achieve this recognition, not only by working within the ISAJE (International Society of Addiction Journal Editors) but, most of all, by its commitment to improvements in the formal aspects and quality of the articles it publishes, making the publication standards clearer and more precise, scrupulously following the Farmington agreements, and by broadening its editorial committee.

Key words: journal, addictions, impact factor, editorial proceedings.
$\mathbf{L}$ a revista Adicciones, órgano científico de Socidrogalcohol, lleva ya quince años publicándose gracias al esfuerzo conjunto de autores, revisores y staff de la revista. Se trata de un proceso editorial consolidado tanto a nivel español como en países de habla hispana, pero ello no supone que nos hayamos quedado sin retos para el futuro, todo lo contrario. Se han mejorado durante estos años tanto los aspectos formales como la calidad de los contenidos, y son alrededor de 1.000 páginas anuales lo que se publica entre los 4 números regulares y los dos monográficos. Entre estos retos que nos quedan está el conseguir indexar la revista en algunas bases de datos internacionales relevantes. Hace unos años conseguimos entrar en Psychinfo que es la base de datos más importante en psicología, gestionada por la American Psychological Association, pero todavía hay bases de datos importantes en las que todavía no hemos con- 
seguido entrar como pueden ser Medline o Current contents.

Current Contents es actualmente la base de datos científica más importante y está gestionada por el Institute for Scientific Information. Analizan alrededor de 12 millones de referencias anuales que corresponden a más de 8.600 revistas y 2.000 libros de todas las ramas del saber, entre las que se encuentran alrededor de 20 que corresponden exclusivamente al campo de las adicciones, todas ellas en lengua inglesa. Cada año se presentan a dicho instituto unas 2.000 revistas de las que sólo alrededor de un $10 \%$ son aceptadas. En este instituto elaboran lo que se llama el 'factor de impacto' que se calcula para cada revista incluida en dicha base de datos a partir del número de citas que en un año han recibido sus artículos de los dos años anteriores, dividido por el número de artículos citados. ¿Por qué nos preocupa no estar incluidos en esta base de datos? Pues no solo por una cuestión narcisista de estar incluidos entre los mejores, sino también por una cuestión práctica, pues hay autores que prefieren siempre que pueden publicar en revistas con factor de impacto -pues ello es importante dentro de su carrera académica- lo cual juega negativamente para las revistas nacionales o de habla no inglesa. Es decir, no estar en esta base de datos genera una situación viciada nada fácil de resolver para las revistas que no están incluidas en la base especialmente si no son inglesas, pues al no conseguir buenos artículos sus posibilidades son menores, mientras que las que ya están tienen más fácil permanecer. La situación además tiene su importancia social pues las revistas especializadas más importantes -todas ellas obviamente de habla inglesa- tienen sus gabinetes de prensa que difunden noticias en lenguaje periodístico sobre sus principales noticias. El resultado de estas prácticas introduce un profundo sesgo. En una revisión de las noticias científicas aparecidas en 1998 en los periódicos españoles de tirada nacional durante medio año aparecen 1.458 noticias sobre ciencia. Ninguna de ellas se basa en resultados publicados en una revista científica española o de habla española (Elias, 1998). Existen direcciones web en ingles donde los periodistas científicos se suscriben y desde donde les proveen de información científica obviamente en inglés y que responde no sólo a criterios científicos sino también comerciales.

En un estudio reciente (Camí, Suñen, Carbó y Coma, 2002) en que se analiza la producción científica española en biomedicina y ciencias de la salud a lo largo del período 1994-2000 el número total de revistas distintas utilizadas por los autores españoles para todo tipo de documentos fue de 2.637, de las que 2590 fueron revistas con documentos en inglés, 52 en francés, 30 en español y 29 en alemán, entre otros idiomas. Entre los documentos que suelen recibir citas el $87 \%$ fueron publicados en inglés, documentos que acumularon el $98,1 \%$ de todas las citas. Las publicaciones en español (12,5\% sobre el total) sólo acumularon el $1,8 \%$ de las citas y el tanto por ciento de estos documentos nunca citados se elevó al $68,1 \%$ de media. Por tanto estamos en una situación de desventaja que interesa claramente mejorar entre todos para evitar el monopolio inglés de la ciencia y que las revistas y publicaciones no inglesas solamente tengan una función educativa y no científica.

Por tanto publicar en función básicamente del factor de impacto introduce importantes problemas incluso dentro del mundo de las revistas en lengua inglesa. Las revistas sobre adicciones tienen cada una de ellas sus peculiaridades y parece bueno que así sea, pero los autores intentan, dentro de esta lógica competitiva basada en el factor de impacto intentar publicar en las revistas de más impacto. Seguramente se hace necesario tomar medidas para corregir el sesgo que introduce en el mundo científico la cuestión del factor de impacto. Parecería que las instituciones de los países no anglosajones -en este caso España- deberían buscar formulas para que el rendimiento académico, por ejemplo, no descansase tanto sobre las publicaciones en revistas de impacto. Existen publicaciones diversas (Buela-Casal, 2003) que analizan esta cuestión del impacto, proponiendo mejoras, pero sobre todo proponiendo una mejor interpretación de lo que significa realmente. En este sentido el factor de impacto de una revista no asegura que todo lo que se publica en ella sea de la misma calidad ni sea igualmente citado por otras revistas.

Pero la revista Adicciones no está en condiciones de reconducir una situación tan compleja y lo que se trata es de hacer una lectura positiva de la realidad en la que se encuentra, sin victimismos y con espíritu de superación. Y este es el reto que asumimos. Mejorar nuestra calidad y nuestro compromiso con la comunidad científica de habla hispana. Es el momento de dar otro fuerte impulso a la revista Adicciones para que la misma tenga un mayor impacto no sólo en nuestro medio sino más allá de nuestras fronteras. Tiene que convertirse en un referente claro y aceptado de la producción científica en el campo de las adicciones.

Nuestra revista forma parte de ISAJE (International Association of Addiction Journal Editors), que es la asociación internacional de editores de revistas científicas en el campo de las adicciones. En la misma están representadas las revistas más importantes a nivel internacional en el campo de las adicciones, publicadas tanto en lengua inglesa como en otros idiomas, como es el caso de En su última reunión en Egham (justo donde se firmó la Carta Magna inglesa), cerca de Londres, a principios de septiembre de este año, y hemos tenido una vez más la oportunidad de intercambiar puntos de vista con otros editores de 
revistas de adicciones de todo el mundo. Algunos aspectos sobre los que ISAJE insiste ya van siendo conocidos por los lectores de Adicciones, como es la insistencia en la adecuación de los artículos al Acuerdo de Farmington (Calafat, 2000) para todo el proceso de revisión y aceptación de artículos en la revista, conflicto de intereses, cuestiones éticas, etc. Pero también nos estamos centrando en mejorar aspectos formales acerca de la presentación de la revista; y, el reforzamiento del equipo directivo con dos editores asociados. Al mismo estamos actualmente en el proceso de reforzar el consejo editorial con la inclusión de especialistas nacionales e internacionales de renombre así como mejorar las normas de publicación, sobre las que haremos un breve comentario más adelante.

Respecto a los autores de los artículos para la revista consideramos que hay varios procesos que tenemos que mejorar, hecho que no solo nos afecta a nosotros como revista, o como profesionales del campo de las adicciones o de las drogodependencias, sino que es una preocupación permanente de las mejores revistas del campo de las adicciones como del resto de los campos científicos. Concretamente, en la citada reunión de ISAJE en Egham, se estuvo de acuerdo en que los aspectos en los que hay que profundizar cara a los autores son cinco. El primero, el proceso de revisión. Los autores tienen que tener claro qué artículos son posibles de aceptar en cada revista y el proceso de revisión de los expertos de los mismos tiene que ser lo más claro posible. Las nuevas normas de Adicciones que se publican en este número pretenden clarificar muchos de estos aspectos tanto para los autores como para los revisores. El segundo es el de la educación a los autores, en el sentido de que deben remitir los artículos siguiendo las normas de publicación de la revista, que los trabajos que remitan a la misma tienen que tener una buena calidad científica, y que se deben adecuar estrictamente a las normas éticas de publicación, como las que recoge el Acuerdo de Farmington (conflicto de intereses, autoría, etc.). El tercero es el de la mejora de la calidad. Conforme una revista científica se va afianzando es necesario mejorar la calidad de la misma; o, a la inversa, al mejorarse la calidad de una revista científica se hace relevante y se afianza en ese campo. Esta es una meta que Adicciones ha tenido desde su fundación y los años indican que poco a poco dicha meta se va cumpliendo etapa a etapa. Pero aún no hemos llegado a la meta, siendo una labor de todos conseguirlo. El cuarto son las cuestiones de copyright. Esto es, un artículo publicado en una revista no puede ser publicado total o parcialmente en otra u otras; cuando se incluye material de otras fuentes, o tablas o figuras, hay que tener los permisos para reproducirlo, etc. Las nuevas normas de publicación de Adicciones indican para los autores guías claras a seguir en este punto. Y, en quinto lugar, eliminar el autoplagio. Este consiste en que un autor utiliza partes importantes de trabajos suyos previos en otro artículo del que afirma que es original. A esto hay que prestar una especial atención porque la ciencia debe ser acumulativa, no repetitiva. Unas normas claras sobre éste y sobre los demás aspectos ya citados facilitarán al autor o autores de los artículos mejorar el contenido de los mismos y la adecuación a los estándares éticos internacionales.

Lo que subyace a todo lo anterior es una apuesta clara por la calidad. Y la calidad exige nuevos estándares. Por ello, en este mismo número aparecen unas nuevas normas de publicación de la revista Adicciones, de modo amplio. También, como es habitual, deben aparecer las mismas en la propia revista de modo abreviado. En dichas normas de publicación se van desgranando algunos de los aspectos ya comentados más atrás. Con las mismas se pretende facilitarle al autor la elaboración de sus manuscritos para enviar a la revista y a los revisores su trabajo de revisión del mismo para que entre unos y otros se mejore el trabajo que se va haciendo en el campo de las adicciones y de las drogodependencias. Como allí se puede apreciar hay algunos cambios que van en la línea de facilitar a los profesionales, clínicos e investigadores el que puedan presentar sus trabajos para publicar en la revista Adicciones de un modo más sencillo y ágil, junto a los formatos ya conocidos hasta ahora en las secciones de la revista. Así, algunas secciones hasta ahora poco utilizadas, como la de artículos cortos, se potenciará a partir de ahora de modo importante. En la sección de artículos cortos pueden presentarse desde casos clínicos, observaciones novedosas, investigaciones en formato breve, etc. Desde aquí ya se anima a que se envíen artículos en este formato, como en el de las demás secciones de la revista. El resto de las secciones se mantienen como hasta ahora, como son las de editorial, artículos originales, artículos de revisión, sección internacional y cartas al director. Estas normas también facilitan un proceso más ágil de envío de manuscritos por parte de los autores al director de la revista y el del proceso de revisión, que dicho sea de paso, es muy bueno a nivel de tiempos en relación con otras revistas científicas. Nuestra idea es mantenerlo así o, incluso, de ser posible, mejorarlo.

Las nuevas normas de publicación incluyen algunos aspectos que es necesario cuidar, como son las cuestiones éticas, aspecto éste que es de una gran preocupación a nivel internacional en el campo de las adicciones y en otros campos. El estar la revista Adicciones dentro del Acuerdo de Farmington, acuerdo que asume y aplica, es una garantía de calidad y de homogenización con otros países de nuestro entorno en el proceso de elaboración, revisión y publicación 
de trabajos científicos. En dichas normas se especifican estos claramente y se dan claras guías para los autores. Dicho acuerdo puede verse como uno de los apéndices de las normas de publicación de la revista Adicciones que aparece en este mismo número, como también ya es conocido por haber sido publicado en un número previo de la revista.

En las nuevas normas de publicación se describen claramente qué aspectos se deben incluir en cada apartado del artículo, en los originales y en los artículos cortos. Respecto al sistema de referencias la revista Adicciones, dada su interdisciplinariedad, mantiene dos sistemas de referencias para que los autores opten por uno de ellos: el del Comité Internacional de Editores de Revistas Médicas, estilo Vancouver, en su última versión del 2001, y el de la American Psychological Association, en su quinta edición, también del año 2001. Como se puede ver en las normas de publicación se indican claros ejemplos de cómo realizar las referencias en uno u otro sistema para facilitar al autor la elaboración de las mismas. A su vez se le indica a los autores cómo acudir directamente a las fuentes (libros o páginas web) para solucionar posibles dudas que les puedan surgir.

La publicación de las normas de publicación se acompañan de tres anexos: el Acuerdo de Farmington; los aspectos que deben estar incluidos en la carta enviada al director de la revista, firmada por todos los autores del manuscrito; y, un listado de comprobación del manuscrito antes de ser enviado para su publicación. Consideramos que este último apéndice puede ser muy útil para los autores. En ellos encontrará algunas soluciones a posibles dudas que puedan tener antes de enviar el artículo para su publicación. Todo lo anterior también lo puede encontrar el autor en formato electrónico dentro del apartado de la revista Adicciones de la web de Socidrogalcohol (www.socidrogalcohol.org). Igualmente, puede enviar el manuscrito por e-mail al director de la revista, con lo que se gana tiempo en el proceso de revisión. Con ello damos un paso más en facilitar el proceso de envío de manuscritos y en el de agilizar todo el proceso de recepción, revisión y posterior de revisión de pruebas y de edición final del manuscrito.

También, por la necesidad de la internacionalización de la revista, y como ya hemos comenzado tímidamente en estos últimos números, se van a publicar en inglés editoriales y artículos cortos, cuando el autor lo haya escrito originariamente en esa lengua, y la traducción del mismo en castellano. Ello facilita la internacionalización de la revista, una mayor difusión de la misma y una mejor penetración internacional. Con ello esperamos poder conseguir en un plazo relativamente breve mejorar nuestra posición para poder entrar en aquellas bases de datos en las que todavía no estamos, lo cual exige un incremento constante de la calidad y mantener la misma en el tiempo.

El poder entrar en las bases de datos más relevantes que le faltan a la revista Adicciones redundará positivamente tanto en los autores que publican en Adicciones, porque sus trabajos serán más conocidos internacionalmente y más citados, como en el propio reconocimiento del campo de las adicciones, del alcoholismo y de las drogodependencias español a nivel internacional. Para nosotros esta es una labor a la que le estamos dedicando y seguiremos dedicando un tiempo especial, junto al resto de las tareas habituales que lleva sacar los números de una revista para que su calidad, contenidos y novedades sean útiles a todos sus lectores y ello redunde en la mejora del conocimiento y en el trabajo cotidiano que realizamos.

También se va a continuar con la publicación de números monográficos sobre sustancias, uno por año, números que han recibido una aceptación excelente entre los lectores de la revista, los profesionales de las drogodependencias y otras personas que se han acercado a este campo. No sólo sirven de reciclaje para todos sino que también constituyen una clara señal de identidad de nuestro campo. Cada número viene a constituir un compendio actualizado de los conocimientos que tenemos sobre todos los aspectos del problema de las drogodependencias: históricos, sociales, médicos, farmacológicos, psicológicos, terapéuticos, preventivos, etc., organizado en torno a una sustancia.

Pero todo esto no podrá llevarse a cabo si los clínicos, profesionales e investigadores no nos envían sus mejores artículos a la revista Adicciones. La nueva estructura de la revista, con sus secciones, permite una mayor flexibilidad para la publicación de los trabajos que envíen los autores siempre que tengan suficiente calidad. A ellos les animamos a que envíen sus manuscritos y los esperamos a partir de ahora. La mejora de una revista y la consecución de una buena calidad es una tarea de todos, comenzando dicha tarea por los autores que nos envían sus trabajos. Es indudable el incremento de la calidad de los trabajos publicados en la revista en los últimos años, como un reflejo de la mejora y profesionalización en el campo de las drogodependencias en nuestro medio. En esta nueva etapa, que consideramos de madurez del campo de las adicciones, del alcoholismo y de las drogodependencias en nuestro medio, esperamos aumentarla aún mucho más. La revista Adicciones, que es sobre todo lo que se publica en sus páginas, es un fiel reflejo de nuestro campo. Por ello, esperamos seguir cumpliendo nuestra misión, como órgano científico de una sociedad científica y profesional, Socidrogalcohol, comprometida con el avance científico y profesional del campo de las adicciones, del alcoholismo y de las drogodependencias. 


\section{REFERENCIAS}

Buela-Casal, G. (2003). Evaluación de la calidad de los artículos y de las revistas científicas: propuesta del factor de impacto ponderado y de un índice de calidad. Psicothema. 15, 23-35.

Calafat, A. (2000). El acuerdo de Farmington Adicciones, 12, 7-10.

Camí, J.; Suñen, E.; Carbó, J.M.; Coma, L. (2002). Producción Científica Española en Biomedicina y Ciencias de la Salud (1994-2000). Informe del Instituto de Salud Carlos III. Recuperado el 20 de octubre de 2003 de http://www.isciii.es/mapabibliometrico

Elias, C. (1998). Influencia de las revistas de impacto en el periodismo científico y en la ciencia actual. Reis, 2 , 123-137. 


\section{SOCIDROCALCOHOL}

\section{SOLICITUD DE INSCRIPCIÓN COMO SOCIO}

Nombre y dos apellidos

Edad.......... Año de nacimiento ....

$\mathrm{DNI} / \mathrm{NIF}$

Tel./Fax.

Domicilio

C.P.

Ciudad y Provincia

Profesión.

Cargo

Centro de trabajo y dirección

Socios que avalan su inscripción (dos).

Nombre

Nombre

En,

de

de.

El solicitante, (Firma)

Sr. Director de,

BANCO o CAJA DE AHORROS

Sucursal

Dirección

C.P.

Ruego a Uds. que hasta nuevo aviso se sirvan atender con cargo a mi Cuenta Corriente/libreta de ahorro $\mathrm{n}^{\circ}$ Entidad.

Oficina D.C los recibos que a mi nombre le sean presentados por,

\section{SOCIDROGALCOHOL}

Les saluda atentamente, (Firma)

En,

de

de

Nombre

NIF

A. SOCIDROGALCOHOL. Sírvanse tomar nota que doy orden a

BANCO o CAJA DE AHORROS

Sucursal. .Dirección

C.P. Ciudad para que sean atendidos con cargo a

mi, C/C o Llbreta $n^{\circ}$. Entidad Oficina D.C.

los recibos que por Cuota de Asociados a SOCIDROGALCOHOL (C.I.F. G58333420), me sean presentados por esa Asociación.

En, a ... de de (Firma)

Nombre

Domicilio

C.P. Ciudad

NOTA: Enviar este impreso íntegro a la Presidencia de SOCIDROGALCOHOL, quien tras tomar los datos oportunos remitirán al Banco o Caja de Ahorros que proceda, la parte superior del mismo. ¡GRACIAS!

SOCIDROGALCOHOL. Vía Augusta, 229 bajos. 08021 BARCELONA. Tel. 9717274 34. Fax: 971213306 • E-mail: secretaria.adicciones@eurociber.es 